\title{
PHONON AND POLARON STATES OF A QUANTUM WELL HETEROSTRUCTURE OF CRYSTALS WITH A HEXAGONAL LATTICE STRUCTURE
}

\author{
V. I. Boichuk, V. A. Borusevych, I. S. Shevchuk \\ Ivan Franko Drohobych State Pedagogical University \\ Department of Theoretical Physics \\ 24 Ivan Franko Str., Drohobych 82100, Ukraine \\ e-mail: vika.borusevych@gmail.com
}

\section{Abstract \\ PHONON AND POLARON STATES OF A QUANTUM WELL HETEROSTRUCTURE OF CRYSTALS WITH A HEXAGONAL LATTICE STRUCTURE}

\section{I. Boichuk, V. A. Borusevych, I. S. Shevchuk}

The article is a theoretical analysis of wave vector dependent energies of confined, interface, half-space, and propagating phonons for symmetric and asymmetric three-layer heterosystems of crystals having a hexagonal lattice structure. The polaron dispersion relation in the $G a N$ crystal and $A l N / G a N / A l N$ double nanosize heterostructure is investigated. All types of polarization vibrations with which an electron interacts are taken into account. The calculations are performed within the finite and infinite barrier models. It is shown that the interface phonon contribution in the polaron energy decreases with increasing nanofilm thickness while that of confined phonons rises. The calculation results of the polaron dispersion relation in the region $k<k_{f}\left(k_{f}=\sqrt{\frac{2 m_{e} \omega}{\hbar}}\right)$ in different polaron wave vector directions with respect to the $c$-axis of the crystal are given. Also, the polaron average speed and effective mass are calculated. The data suggest an effective enhancement of the electron-phonon interaction with lowering system dimensions number.

Keywords: nanoheterostructure, quantum well, electron-phonon interaction, polaron, perturbation theory, variational method

\section{Анотація \\ ФОНОННІ ТА ПОЛЯРОННІ СТАНИ НАНОГЕТЕРОСТРУКТУРИ З КВАНТОВОЮ ЯМОЮ КРИСТАЛІВ ГЕКСАГОНАЛЬНОЇ СИМЕТРІЇ.}

\section{В. І. Бойчук, В. А. Борусевич, І. С. Шевиук}

В даній роботі теоретично досліджено залежності енергії обмежених (confined), міжповерхневих (interface), напівобмежених (half-space) фононів та фононів, що поширюються (propagating phonons), від хвильового вектора для симетричних, та несиметричних тришарових наногетеросистем кристалів гексагональної симетрії. Досліджено також закон дисперсії поляронів у кристалі $G a N$ та у подвійній наногетероструктурі $A l N / G a N / A l N$. Враховано всі типи поляризаційних коливань, з якими взаємодіє електрон. Проведено обчислення в рамках моделі скінченного та нескінченного розриву зон. Показано, що зі збільшенням товщини наноплівки внесок міжповерхневих фононів у енергію полярона зменшується, а 
обмежених фононів збільшується. Подано результати обчислень закону дисперсії полярона в області $k<k_{f}\left(k_{f}=\sqrt{\frac{2 m_{e} \omega}{\hbar}}\right)$ при різних напрямках хвильового вектора полярона відносно С-осі кристалу. Проведено обчислення середньої швидкості та ефективної маси полярона. Показано, що із зменшенням розмірності системи виникає ефективне підсилення електрон-фононної взаємодії.

Ключові слова: наногетероструктура, квантова яма, електрон-фононна взаємодія, полярон, теорія збурень, варіаційний метод

\section{Аннотация \\ ФОНОННЫЕ И ПОЛЯРОННЫЕ СОСТОЯНИЯ НАНОГЕТЕРОСТРУКТУРЫ С КВАНТОВОЙ ЯМОЙ КРИСТАЛЛОВ ГЕКСАГОНАЛЬНОЙ СИММЕТРИИ.}

\section{В. И. Бойчук, В. А. Борусевич, И.С. Шевчук}

В данной работе теоретически исследованы зависимости энергии ограниченных (confined), межповерхностных (interface), полуограниченных (half-space) фононов и фононов, которые распространяются (propagating phonons), от волнового вектора для симметричных, и несимметричных трехслойных наногетеросистем кристаллов гексагональной симметрии. Исследован также закон дисперсии поляронов в кристалле $G a N$ и в двойной наногетероструктуре $A l N / G a N / A l N$. Учтены все типы поляризационных колебаний, с которыми взаимодействует электрон. Проведены вычисления в рамках модели конечного и бесконечного разрыва зон. Показано, что с увеличением толщины нанопленки взнос межповерхностных фононов в энергию полярона уменьшается, а ограниченных фононов увеличивается. Представлены результаты вычислений закона дисперсии полярона в области $k<k_{f}\left(k_{f}=\sqrt{\frac{2 m_{e} \omega}{\hbar}}\right)$ при разных направлениях волнового вектора полярона относительно С-оси кристалла. Проведены вычисления средней скорости и эффективной массы полярона. Показано, что с уменьшением размерности системы возникает эффективное усиление электрон-фононного взаимодействия.

Ключевые слова: наногетероструктура, квантовая яма, электрон-фононное взаимодействие, полярон, теория возмущений, вариационный метод

\section{Introduction}

In the last two decades main results in physics of semiconductor heterostructures are related to a considerable degree to investigations of the electron-optical phonon interaction. The electronphonon interaction is decisive in the analysis of electron scattering, energy exchange between hot electrons and the lattice, polaron effects etc. In most reports this interaction is based on the dielectric continuum model (DCM). The use of DCM makes it possible to obtain relatively simple analytical expressions for different physical quantities [1]. In addition, the results received within the framework of the DC model are in good agreement with the data of detail microscopic calculations $[2,3]$ and experiment $[4,5]$.

The electron-phonon interaction is investigated sufficiently well in different low-dimensional systems: quasi-two-dimensional heterostructures, quantum wires and quantum dots made from optically isotropic materials. However, in recent years anisotropic materials such as $\mathrm{ZnO}, \mathrm{GaN}, \mathrm{AlN}$, and InN draw physisists' attention because of their application in electronics. Therefore, in the dielectric continuum model optical phonons and their interaction with an electron in heterostructures with one [7] and two interfaces [7-9], infinite superlattices [10], and also different multilayer heterostructures $[11,12]$ are examined. Theoretical researches stimulated a number of experimental works which involved the measurement of the Raman spectra of heterosystems [13-16].

As a rule, theoretical studies consider symmetric heterosystems where a nanofilm of one crystal is 
adjacent to identical media (e.g., AlN/GaN/AlN). Such a structure is a partial case of the asymmetric three-layer heterosystem in which semi-confined media are different.

The purpose of the work is a theoretical analysis of the polarization phonon and polaron dispersion relations in the double nanoheterostructure of hexagonal lattice structure crystals.

\section{Statement of the problem. General formulas}

A three-layer planar heterostructure with a nanofilm of thickness $\mathrm{L}$ between two semi-infinite media is considered (Fig. 1). The coordinate system is chosen in such a way that the crystallographic axis $\mathrm{C}$ coincides with the $\mathrm{z}$-axis. The separation boundaries of the heterosystem are given by

$$
|z|=L / 2 \text {. }
$$

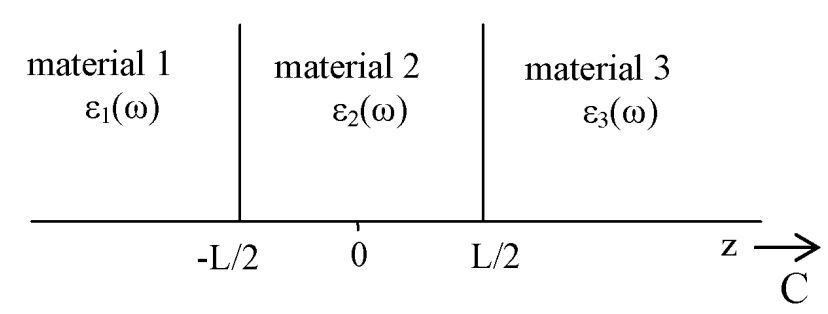

Fig. 1

Since the system contains no free charges the potential of polarization oscillations is defined from the Laplace equation:

$$
\begin{gathered}
{\left[\varepsilon_{z}(\omega) \frac{\partial^{2}}{\partial z^{2}}+\varepsilon_{\perp}(\omega) \nabla_{\vec{\rho}}\right] \Phi(\vec{r})=0,} \\
\varepsilon_{z}(\omega)=\varepsilon_{z}^{\infty} \frac{\omega^{2}-\omega_{L z}^{2}}{\omega^{2}-\omega_{T z}^{2}}, \varepsilon_{\perp}(\omega)=\varepsilon_{\perp}^{\infty} \frac{\omega^{2}-\omega_{L t}^{2}}{\omega^{2}-\omega_{T t}^{2}},
\end{gathered}
$$

where $\vec{\rho}=x \vec{i}+y \vec{j}$,

$\omega_{L z}, \omega_{T z}, \omega_{L t}, \omega_{T t}$ are characteristic frequencies of $\mathrm{A}_{1}(\mathrm{LO})-, \mathrm{A}_{1}(\mathrm{TO})-, \mathrm{E}_{1}(\mathrm{LO})-, \mathrm{E}_{1}(\mathrm{TO})-$ modes, $\varepsilon_{z}^{\infty}$ and $\varepsilon_{\perp}^{\infty}$ are high-frequency dielectric permittivities. Due to translational symmetry of the heterostructure in the directions XOY, the potential $\Phi(\vec{r})$ can be represented by the Fourier series:

$$
\Phi(\vec{r})=\sum_{\vec{q}} \Phi(z) e^{i \vec{q} \vec{\rho}} .
$$

Substitution (2) in Eq. (1) makes it possible to get the expression for $\Phi(z)$

$$
\left(q^{2} \varepsilon_{\perp}-\varepsilon_{z} \frac{\partial^{2}}{\partial z^{2}}\right) \Phi(z)=0 .
$$

If continuity of the potential and normal component of the induction vector is taken into account $\left(\vec{D}=\varepsilon_{\perp}(\omega) \vec{E}_{\perp}+\varepsilon_{z}(\omega) E(z) \vec{k}\right)$, one can obtain the conditions

$$
\begin{gathered}
\Phi_{1}(-L / 2)=\Phi_{2}(-L / 2), \\
\Phi_{2}(L / 2)=\Phi_{3}(L / 2), \\
\left.\varepsilon_{1 z} \frac{\partial \Phi_{1}}{\partial z}\right|_{z=-L / 2}=\left.\varepsilon_{2 z} \frac{\partial \Phi_{2}}{\partial z}\right|_{z=-L / 2}, \\
\left.\varepsilon_{2 z} \frac{\partial \Phi_{2}}{\partial z}\right|_{z=L / 2}=\left.\varepsilon_{3 z} \frac{\partial \Phi_{3}}{\partial z}\right|_{z=L / 2} .
\end{gathered}
$$

In the wurtzite-based crystals there exist two phonon types: ordinary and extraordinary. The first phonon type is quanta of transverse oscillations non-interacting with an electron. Extraordinary phonons are characterized by the fact that $\vec{E}$ is parallel to $\vec{Q}$ and they interact with electrons, e.g., by creating polaron states. Therefore, we further consider only the properties of extraordinary phonons.

From Eq. (1) it is easy to get the dispersion relation of extraordinary phonons in a bulk crystal,

$$
\varepsilon_{z}(\omega) Q_{z}^{2}+\varepsilon_{\perp}(\omega) q^{2}=0 .
$$

The phonon energy spectrum in the heterostructure is based on the analysis of Eq. (3). It is seen that a general solution of the equation is expressed

$$
\Phi(z)=A e^{\chi z}+B e^{-\chi z}, \chi=\sqrt{\frac{\varepsilon_{\perp}(\omega)}{\varepsilon_{z}(\omega)}} q .
$$

If $\varepsilon_{\perp} \varepsilon_{z}<0$, one obtains solutions for oscillating phonon waves. And if $\varepsilon_{\perp} \varepsilon_{z}>0$, phonon waves are decaying ( $\chi$ is a real quantity).

For the heterostructures of crystals with a hexagonal lattice structure there can exist confined, interface half-space, and propagating phonons.

Confined polarization phonons are characterized with oscillating solutions for $\Phi(z)$ inside $(-L / 2 \leq z \leq L / 2)$ and decaying solutions outside the nanofilm: $|z|>L / 2$. Thus, in order for confined polarization vibrations to exist, the following conditions must be satisfied:

$$
\varepsilon_{2 \perp} \varepsilon_{2 z}<0, \varepsilon_{1 \perp} \varepsilon_{1 z}>0, \varepsilon_{3 \perp} \varepsilon_{3 z}>0
$$

If we introduce the notation 


$$
\Phi(z)= \begin{cases}\Phi_{1}(z), & z<-L / 2 \\ \Phi_{2}(z), & |z| \leq L / 2 \\ \Phi_{3}(z), & z>L / 2\end{cases}
$$
ten

the quantities $\Phi_{1}(z), \Phi_{2}(z), \Phi_{3}(z)$ are writ-

$$
\begin{gathered}
\Phi_{1}(z)=A_{1} e^{\chi_{1}(z+L / 2)}, \chi_{1}=\sqrt{\frac{\varepsilon_{1 \perp}}{\varepsilon_{1 z}}} q, \\
\Phi_{2}(z)=A_{2} \cos \left(\kappa_{2} z\right)+B_{2} \sin \left(\kappa_{2} z\right), \\
\kappa_{2}=\sqrt{\left|\frac{\varepsilon_{2 \perp}}{\varepsilon_{2 z}}\right|} q, \\
\Phi_{3}(z)=B_{1} e^{-\chi_{3}(z-L / 2)}, \chi_{3}=\sqrt{\frac{\varepsilon_{3 \perp}}{\varepsilon_{3 z}}} q .
\end{gathered}
$$

By substituting (9)-(11) in Eq. (4), one can obtain the system of homogeneous equations over coefficients $A_{1}, A_{2}, B_{2}, B_{3}$. Since these coefficients are different from zero, we receive the equation for the wave vector $q$ and frequency $\omega$ :

$$
\begin{gathered}
\varepsilon_{2 z}\left(\sqrt{\frac{\varepsilon_{1 \perp}}{\varepsilon_{1 z}}} \varepsilon_{1 z}+\sqrt{\frac{\varepsilon_{3 \perp}}{\varepsilon_{3 z}}} \varepsilon_{3 z}\right) \times \\
\times \sqrt{\frac{\varepsilon_{2 \perp}}{\varepsilon_{2 z}} \mid} \cos \left(L q \sqrt{\left|\frac{\varepsilon_{2 \perp}}{\varepsilon_{2 z}}\right|}\right)+ \\
+\left(\sqrt{\frac{\varepsilon_{1 \perp}}{\varepsilon_{1 z}}} \varepsilon_{1 z} \sqrt{\frac{\varepsilon_{3 \perp}}{\varepsilon_{3 z}}} \varepsilon_{3 z}-\frac{\varepsilon_{2 \perp} \varepsilon_{2 z} \operatorname{sign}\left(\varepsilon_{2 z}\right)}{\operatorname{sign}\left(\varepsilon_{2 \perp}\right)}\right) \times \\
\times \sin \left(L q \sqrt{\left|\frac{\varepsilon_{2 \perp} \mid}{\varepsilon_{2 z}}\right|}\right)=0 .
\end{gathered}
$$

In case of a symmetric heterostructure $\left(\varepsilon_{1 z}=\varepsilon_{3 z}\right.$, $\left.\varepsilon_{1 \perp}=\varepsilon_{3 \perp}\right)$ phonon states divide into two types: symmetric $\left(\Phi_{2}(z)=A_{2} \cos \left(\kappa_{2} z\right)\right)$ and antisymmetric $\left(\Phi_{2}(z)=B_{2} \sin \left(\kappa_{2} z\right)\right)$. For symmetric confined phonons the dispersion relation looks as

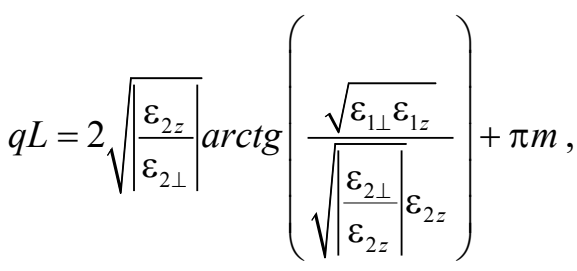

$$
\begin{aligned}
& m=0,1,2,3, \ldots .
\end{aligned}
$$

The dispersion relation of antisymmetric phonons has the form

$$
q L=\pi m-2 \sqrt{\frac{\varepsilon_{2 z} \mid}{\varepsilon_{2 \perp} \mid}} \operatorname{arctg}\left(\frac{\sqrt{\frac{\varepsilon_{2 \perp} \mid}{\varepsilon_{2 z} \mid}} \mid \varepsilon_{2 z}}{\sqrt{\varepsilon_{1 \perp} \varepsilon_{1 z}}}\right),
$$$$
m=0,1,2,3, \ldots .
$$

Eqs. (13) and (14) being partial cases of Eq. (12) are identical to equations obtained in [9]. These equations are further simplified for a symmetric heterostructure of crystals of a cubic lattice structure and correspond to the equations in [17].

Interface phonons are described with decaying solutions at both interfaces. Thus, the following inequalities should be valid:

$$
\begin{gathered}
\varepsilon_{2 \perp} \varepsilon_{2 z}>0, \varepsilon_{1 \perp} \varepsilon_{1 z}>0, \varepsilon_{3 \perp} \varepsilon_{3 z}>0, \\
\varepsilon_{1 z} \varepsilon_{2 z}<0, \varepsilon_{2 z} \varepsilon_{3 z}<0 .
\end{gathered}
$$

The potentials $\Phi_{1}(z)$ and $\Phi_{3}(z)$ are expressed by (9) and (11), while

$$
\begin{gathered}
\Phi_{2}(z)=A_{2} \operatorname{ch}\left(\chi_{2} z\right)+B_{2} \operatorname{sh}\left(\chi_{2} z\right), \\
\chi_{2}=\sqrt{\frac{\varepsilon_{2 \perp}(\omega)}{\varepsilon_{2 z}(\omega)}} q .
\end{gathered}
$$

In this case the dispersion relation looks as follows:

$$
\begin{aligned}
& \varepsilon_{2 z}\left(\sqrt{\frac{\varepsilon_{1 \perp}}{\varepsilon_{1 z}}} \varepsilon_{1 z}+\sqrt{\frac{\varepsilon_{3 \perp}}{\varepsilon_{3 z}}} \varepsilon_{3 z}\right) \sqrt{\frac{\varepsilon_{2 \perp}}{\varepsilon_{2 z}}} \cos \left(L q \sqrt{\frac{\varepsilon_{2 \perp}}{\varepsilon_{2 z}}}\right)+ \\
& +\left(\sqrt{\frac{\varepsilon_{1 \perp}}{\varepsilon_{1 z}}} \varepsilon_{1 z} \sqrt{\frac{\varepsilon_{3 \perp}}{\varepsilon_{3 z}}} \varepsilon_{3 z}+\varepsilon_{2 \perp} \varepsilon_{2 z}\right) \operatorname{sh}\left(L q \sqrt{\frac{\varepsilon_{2 \perp}}{\varepsilon_{2 z}}}\right)=0
\end{aligned}
$$

Like in the case of confined phonons for the symmetric heterosystem $\left(\varepsilon_{1 z}=\varepsilon_{3 z}, \varepsilon_{1 \perp}=\varepsilon_{3 \perp}\right)$, interface phonons are divided into symmetric ( $\left.B_{2}=0\right)$ and antisymmetric $\left(A_{2}=0\right)$. The dispersion relation of symmetric interface phonons has the form

$$
q L=2 \sqrt{\frac{\varepsilon_{2 z}(\omega)}{\varepsilon_{2 \perp}(\omega)}} \operatorname{arcth}\left(\frac{\sqrt{\varepsilon_{1 \perp}(\omega) \varepsilon_{1 z}(\omega)}}{\sqrt{\varepsilon_{2 \perp}(\omega) \varepsilon_{2 z}(\omega)}}\right),
$$

and for antisymmetric phonons it can be presented

$$
q L=2 \sqrt{\frac{\varepsilon_{2 z}(\omega)}{\varepsilon_{2 \perp}(\omega)}} \operatorname{arcth}\left(\frac{\sqrt{\varepsilon_{2 \perp}(\omega) \varepsilon_{2 z}(\omega)}}{\sqrt{\varepsilon_{1 \perp}(\omega) \varepsilon_{1 z}(\omega)}}\right) .
$$

Equations (18) and (19) are reduced to analogous equations in [9], and for cubic crystals to those in [17]. 
Half-space phonons appear under the condition when polarization waves are decaying inside the nanofilm and oscillating in the half-spaces $|z| \geq L / 2$ :

$$
\varepsilon_{1 \perp} \varepsilon_{1 z}<0, \varepsilon_{3 \perp} \varepsilon_{3 z}<0, \varepsilon_{2 \perp} \varepsilon_{2 z}>0 .
$$

In this case it is necessary to consider two types of polarization vibrations:

a) quasi-symmetric:

$$
\begin{gathered}
\Phi_{1}(z)=A_{1} \cos \left(\kappa_{1} z\right), \\
\Phi_{2}(z)=A_{2} \operatorname{ch}\left(\chi_{2} z\right)+B_{2} \operatorname{sh}\left(\chi_{2} z\right), \\
\Phi_{3}(z)=A_{3} \cos \left(\kappa_{3} z\right), \\
\kappa_{i}=\sqrt{\mid \frac{\varepsilon_{i \perp}(\omega) \mid}{\varepsilon_{i z}(\omega) \mid} q, i=1,3, \chi_{2}=\sqrt{\frac{\varepsilon_{2 \perp}(\omega)}{\varepsilon_{2 z}(\omega)}} q ;}
\end{gathered}
$$

b) quasi-antisymmetric:

$$
\begin{gathered}
\Phi_{1}(z)=B_{1} \sin \left(\kappa_{1} z\right), \\
\Phi_{2}(z)=A_{2}^{\prime} \operatorname{ch}\left(\chi_{2} z\right)+B_{2}^{\prime} \operatorname{sh}\left(\chi_{2} z\right), \\
\Phi_{3}(z)=B_{3} \sin \left(\kappa_{3} z\right) .
\end{gathered}
$$

For first type phonons the dispersion relation is written

$$
\begin{gathered}
\varepsilon_{2 z} \cos \left(q \frac{L}{2} \sqrt{\frac{\varepsilon_{1 \perp}}{\varepsilon_{1 z}} \mid}\right)\left(\sqrt{\frac{\varepsilon_{2 \perp}}{\varepsilon_{2 z}}} \varepsilon_{3 z} \sqrt{\left|\frac{\varepsilon_{3 \perp}}{\varepsilon_{3 z}}\right|} \times\right. \\
\times \cosh \left(q L \sqrt{\frac{\varepsilon_{2 \perp}}{\varepsilon_{2 z}}}\right) \sin \left(q \frac{L}{2} \sqrt{\left|\frac{\varepsilon_{3 \perp}}{\varepsilon_{3 z}}\right|}\right)+ \\
+\varepsilon_{2 \perp} \cos \left(q \frac{L}{2} \sqrt{\left|\frac{\varepsilon_{3 \perp}}{\varepsilon_{3 z}}\right|}\right) \operatorname{sh}\left(q L \sqrt{\frac{\varepsilon_{2 \perp}}{\varepsilon_{2 z}}}\right)+ \\
+\varepsilon_{1 z} \sqrt{\left|\frac{\varepsilon_{1 \perp}}{\varepsilon_{1 z}}\right|} \sin \left(q \frac{L}{2} \sqrt{\left.\left|\frac{\varepsilon_{1 \perp}}{\varepsilon_{1 z}}\right|\right) \times}\right) \\
\times\left(\sqrt{\frac{\varepsilon_{2 \perp}}{\varepsilon_{2 z}}} \varepsilon_{2 z} \cos \left(q \frac{L}{2} \sqrt{\left|\frac{\varepsilon_{3 \perp}}{\varepsilon_{3 z}}\right|}\right) \operatorname{ch}\left(q L \sqrt{\frac{\varepsilon_{2 \perp}}{\varepsilon_{2 z}}}\right)+\right. \\
+\varepsilon_{3 z} \sqrt{\left|\frac{\varepsilon_{3 \perp}}{\varepsilon_{3 z}}\right|} \sin \left(q \frac{L}{2} \sqrt{\left|\frac{\varepsilon_{3 \perp}}{\varepsilon_{3 z}}\right|}\right) \operatorname{sh}\left(q L \sqrt{\frac{\varepsilon_{2 \perp}}{\varepsilon_{2 z}}}\right)=0 .
\end{gathered}
$$

Quasi-antisymmetric phonons are described by the following dispersion relation:

$$
\begin{aligned}
& \varepsilon_{3 z} \sqrt{\left|\frac{\varepsilon_{3 \perp}}{\varepsilon_{3 z}}\right|} \cos \left(q \frac{L}{2} \sqrt{\frac{\varepsilon_{3 \perp}}{\varepsilon_{3 z}} \mid}\right)\left(\sqrt{\frac{\varepsilon_{2 \perp}}{\varepsilon_{2 z}}} \varepsilon_{2 z} \times\right. \\
& \times \cosh \left(q L \sqrt{\frac{\varepsilon_{2 \perp}}{\varepsilon_{2 z}}}\right) \sin \left(q \frac{L}{2} \sqrt{\frac{\varepsilon_{1 \perp}}{\varepsilon_{1 z}}}\right)- \\
& -\varepsilon_{1 z} \sqrt{\left|\frac{\varepsilon_{1 \perp}}{\varepsilon_{1 z}}\right|} \cos \left(q \frac{L}{2} \sqrt{\left|\frac{\varepsilon_{1 \perp}}{\varepsilon_{1 z}}\right|}\right) \operatorname{sh}\left(q L \sqrt{\frac{\varepsilon_{2 \perp}}{\varepsilon_{2 z}}}\right)+ \\
& +\varepsilon_{2 z} \sin \left(q \frac{L}{2} \sqrt{\left.\mid \frac{\varepsilon_{3 \perp}}{\varepsilon_{3 z}}\right)}\right)\left(\varepsilon_{1 z} \sqrt{\frac{\varepsilon_{2 \perp}}{\varepsilon_{2 z}}} \sqrt{\left|\frac{\varepsilon_{1 \perp}}{\varepsilon_{1 z}}\right|} \times\right. \\
& \times \cos \left(q \frac{L}{2} \sqrt{\frac{\varepsilon_{1 \perp}}{\varepsilon_{1 z}}}\right) \operatorname{ch}\left(q L \sqrt{\frac{\varepsilon_{2 \perp}}{\varepsilon_{2 z}}}\right)- \\
& -\varepsilon_{2 \perp} \sin \left(q \frac{L}{2} \sqrt{\frac{\varepsilon_{1 \perp}}{\varepsilon_{1 z}} \mid}\right) \operatorname{sh}\left(q L \sqrt{\frac{\varepsilon_{2 \perp}}{\varepsilon_{2 z}}}\right)=0 .
\end{aligned}
$$

If the nanoheterosystem is symmetric, then quasi-symmetric phonons become symmetric $\left(B_{2}=0\right)$, and quasi-symmetric ones become antisymmetric $\left(A_{2}^{\prime}=0\right)$. Then the dispersion relations are

$$
\begin{gathered}
q L=\pi m-2 \sqrt{\left|\frac{\varepsilon_{1 z}}{\varepsilon_{1 \perp}}\right|} \times \\
\times \operatorname{arctg}\left\{\frac{\sqrt{\varepsilon_{2 \perp} \varepsilon_{2 z}}}{\varepsilon_{1 z} \sqrt{\left|\frac{\varepsilon_{1 \perp}}{\varepsilon_{1 z}}\right|}} \operatorname{th}\left(\sqrt{\frac{\varepsilon_{2 \perp}}{\varepsilon_{2 z}}} \frac{q L}{2}\right)\right\}
\end{gathered}
$$

for symmetric phonons and

$$
\begin{gathered}
q L=\pi m+2 \sqrt{\left|\frac{\varepsilon_{1 z}}{\varepsilon_{1 \perp}}\right|} \times \\
\times \operatorname{arctg}\left\{\frac{\varepsilon_{1 z} \sqrt{\left|\frac{\varepsilon_{1 \perp}}{\varepsilon_{1 z}}\right|}}{\sqrt{\varepsilon_{2 \perp} \varepsilon_{2 z}}} t h\left(\sqrt{\frac{\varepsilon_{2 \perp}}{\varepsilon_{2 z}}} \frac{q L}{2}\right)\right\}
\end{gathered}
$$

for asymmetric phonons $(m=0,1,2,3, \ldots)$. Like for confined and interface phonons the dispersion relations (24) and (25) are identical to those obtained in [9]. Eqs. (22) and (23) are more general.

Propagating phonons are described by oscillating solutions in all media because the following conditions must be valid: $\varepsilon_{i z} \varepsilon_{i \perp}<0, \quad i=1,2,3$. Be- 
cause of absence of decaying the potential $\Phi(\vec{r})$ is different from zero even for $|z|=\infty$. One should use additional conditions to obtain the dispersion relation of these phonons. It can be proved [1] that without regard for delaying effects for propagating waves the wave vector and the electric field vector are co-linear $(\vec{Q} \times \vec{E}=0)$ :

$$
Q_{z} \frac{\partial \Phi(\vec{r})}{\partial \rho}-q \frac{\partial \Phi(\vec{r})}{\partial z}=0 .
$$

On the basis of this equation on can get the conditions at the interfaces

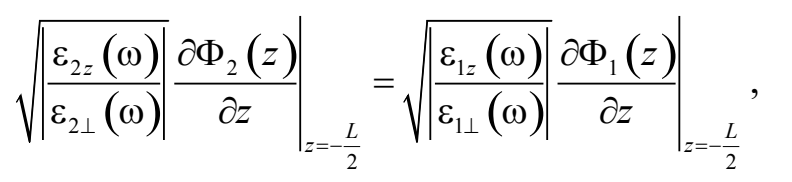$$
\left.\sqrt{\mid \frac{\varepsilon_{2 z}(\omega)}{\varepsilon_{2 \perp}(\omega) \mid}} \frac{\partial \Phi_{2}(z)}{\partial z}\right|_{z=\frac{L}{2}}=\left.\sqrt{\mid \frac{\varepsilon_{3 z}(\omega)}{\varepsilon_{3 \perp}(\omega) \mid}} \frac{\partial \Phi_{3}(z)}{\partial z}\right|_{z=\frac{L}{2}} .
$$

The potential $\Phi(z)$ in different media is given by

$$
\Phi_{i}(z)=A_{i} \cos \left(\kappa_{i} z\right)+B_{i} \sin \left(\kappa_{i} z\right), i=1,2,3,
$$

$$
\kappa_{i}=\sqrt{\left|\frac{\varepsilon_{i \perp}}{\varepsilon_{i z}}\right|} q .
$$

With regard for all conditions at the interfaces, we have obtained the system of algebraic equations which is homogeneous and has non-trivial solutions when its determinant equals zero. Thus, the dispersion relation is written

$$
\frac{\left(\varepsilon_{1 z} \sqrt{\left|\varepsilon_{1 \perp} \varepsilon_{2 z}\right|}-\varepsilon_{2 z} \sqrt{\left|\varepsilon_{1 z} \varepsilon_{2 \perp}\right|}\right)\left(\varepsilon_{2 z} \sqrt{\left|\varepsilon_{2 \perp} \varepsilon_{3 z}\right|}-\varepsilon_{3 z} \sqrt{\left|\varepsilon_{2 z} \varepsilon_{3 \perp}\right|}\right) \sin \left(q L \sqrt{\frac{\varepsilon_{2 \perp} \mid}{\varepsilon_{2 z} \mid}}\right)}{\sqrt{\left|\varepsilon_{1 z} \varepsilon_{3 z}\right|}\left|\varepsilon_{2 z}\right|}=0 .
$$

Forsymmetric three-layer heterosystem $\left(\kappa_{1}=\kappa_{3}\right)$ there are two types of propagating phonons: symmetric -

$$
\begin{gathered}
\Phi_{1}(z)=A_{1} \cos \left(\kappa_{1} z\right), \\
\Phi_{2}(z)=A_{2} \cos \left(\kappa_{2} z\right)+B_{2} \sin \left(\kappa_{2} z\right), \\
\Phi_{3}(z)=A_{3} \cos \left(\kappa_{1} z\right)
\end{gathered}
$$

and antisymmetric -

$$
\begin{gathered}
\Phi_{1}(z)=B_{1}^{\prime} \sin \left(\kappa_{1} z\right), \\
\Phi_{2}(z)=A_{2}^{\prime} \cos \left(\kappa_{2} z\right)+B_{2}^{\prime} \sin \left(\kappa_{2} z\right), \\
\Phi_{3}(z)=B_{3}^{\prime} \sin \left(\kappa_{1} z\right) .
\end{gathered}
$$

By taking account of conditions at the interfaces one can get the dispersion relations for symmetric and antisymmetric phonons. There is a non-dispersive phonon mode the frequency of which is determined by the equation

$$
\varepsilon_{1 z} \sqrt{\frac{\varepsilon_{2 z}}{\varepsilon_{2 \perp}} \mid}-\varepsilon_{2 z} \sqrt{\left|\frac{\varepsilon_{1 z}}{\varepsilon_{1 \perp}}\right|}=0 .
$$

And also the dispersion relation is

$$
q L=\pi \sqrt{\mid \frac{\varepsilon_{1 z}}{\varepsilon_{1 \perp} \mid} m},
$$

where $m=2,4,6, \ldots$ is for symmetric, and $m=1,3,5, \ldots$ is for asymmetric phonons. Equations (31) and (32) are identical to those obtained in [9]. As shown above, this phonon type does not exist in every heterosystem. In heterosystems of cubic crystals these phonons do not appear. Only if heterosystem crystals are of hexagonal lattice structure, then Eqs. (28), (31), (32) describing these phonons can have solutions when the required conditions concerning dielectric functions are satisfied.

In order to research polaron states we consider the Hamiltonian of the phonon system interacting with an electron:

$$
\hat{\mathrm{H}}=\hat{\mathrm{He}}+\hat{\mathrm{H}} \mathrm{ph}+\hat{\mathrm{H}} \text { int } .
$$

In the effective mass approximation $\hat{\mathrm{He}}$ for oneaxis crystal has the form

$$
\begin{gathered}
\hat{\mathrm{He}}=-\frac{\hbar^{2}}{2 m_{\perp}} \nabla_{\vec{\rho}}^{2}-\frac{\hbar^{2}}{2 m_{\|}} \frac{\partial^{2}}{\partial z^{2}}+V, \\
\nabla_{\rho}^{2}=\frac{\partial^{2}}{\partial x^{2}}+\frac{\partial^{2}}{\partial y^{2}},
\end{gathered}
$$

where $m_{\perp}$ and $m_{\|}$are electron effective masses in the perpendicular and parallel directions to the $C-$ axis, $V$ is the electron potential energy. For a bulk crystal $V=0$. 
The phonon energy operator in the occupation number representation is written

$$
\hat{H}_{p h}=\sum_{\overrightarrow{\mathrm{Q}}, v} \hbar \omega_{v}(\vec{q})\left(b_{\overrightarrow{\mathrm{Q}}, v}^{+} b_{\overrightarrow{\mathrm{Q}}, v}+1 / 2\right),
$$

where $b_{\overrightarrow{\mathrm{Q}}, v}^{+}$is a phonon birth operator with the wave vector $\vec{Q}$ of the phonon mode $v$.

Polarization phonon frequency $\omega$ is found from dispersion relation (5). For the wurtzites under consideration conditions [9] are fulfilled:

$$
\left|\omega_{L t}-\omega_{L z}\right|,\left|\omega_{T t}-\omega_{T z}\right|<<\left|\omega_{L t}-\omega_{T t}\right|,\left|\omega_{L z}-\omega_{T z}\right| .
$$

If we account for $q=\mathrm{Q} \cdot \sin (\theta)$ and $\mathrm{Q}_{z}=\mathrm{Q} \cdot \cos (\theta)$, then from Eq. (5) under conditions (36) we can get two solutions for $\omega$ :

$$
\begin{gathered}
\omega_{l}^{2}=\omega_{L z}^{2} \cos ^{2} \theta+\omega_{L t}^{2} \sin ^{2} \theta, \\
\omega_{t}^{2}=\omega_{T z}^{2} \sin ^{2} \theta+\omega_{T t}^{2} \cos ^{2} \theta .
\end{gathered}
$$

The first solution can be referred to as frequency of mainly longitudinal modes, while the second one as frequency of mainly transverse modes. The electron-phonon interaction operator for a bulk crystal can be given by $[7,9]$

$$
\hat{H}_{\text {int }}=\sum_{\overrightarrow{\mathrm{Q}}} V^{(1)}(\overrightarrow{\mathrm{Q}}) \cdot V^{(2)}(\overrightarrow{\mathrm{Q}}, \mathrm{z})^{i \vec{q} \overrightarrow{\mathrm{\rho}}}\left(b_{\overrightarrow{\mathrm{Q}}}+b_{-\overrightarrow{\mathrm{Q}}}^{+}\right),
$$

where $V^{(1)}=\frac{C}{\sqrt{S \tilde{L}} \mathrm{Q}} \frac{1}{\frac{\partial}{\partial \omega}\left(\varepsilon_{\perp}(\omega) \sin ^{2} \theta+\varepsilon_{z}(\omega) \cos ^{2} \theta\right)}$,

$$
\begin{gathered}
V^{(2)}(\overrightarrow{\mathrm{Q}}, \mathrm{z})=e^{i \mathrm{Q}_{\mathrm{Z}} z}, \\
C=\left(4 \pi \hbar e^{2}\right)^{1 / 2}, \mathrm{Q}^{2}=q^{2}+\mathrm{Q}_{\mathrm{Z}}^{2} .
\end{gathered}
$$

$S \tilde{L}$ is the crystal volume, the derivative over frequency is taken at the point $\omega=\omega_{l}$.

It easy to make sure that in the case

$$
\begin{gathered}
\omega_{L t}=\omega_{L z}, \omega_{T t}=\omega_{T z}, \\
\varepsilon_{\perp}^{\infty}=\varepsilon_{z}^{\infty}
\end{gathered}
$$

equations (37)-(39) transfer to the well-known formulas for a cubic lattice structure crystal [18, 19].

For a hexagonal symmetry heterostructure in which two semi-infinite crystals are adjacent to a nanofilm (double heterosystem), there exist four types of optical phonon modes. These modes are referred to - like those for heterosystems of zinc blende - based crystals - interface, confined, half-space. Moreover, generally speaking, there can be a new phonon type in the system (compared to heterostructures of cubic lattice structure crystals) i.e., phonons that propagate in the medium (propagating phonons). Analogously to the case of cubic lattice structure crystal heterosystem, the operator of the electron interaction with different modes can be represented as a sum of symmetric and antisymmetric parts, that is for all the mentioned types there also exist symmetric and antisymmetric phonons.

In general, the electron-phonon interaction operator is a sum of eight terms, each of them is given by

$$
\hat{H}=\sum_{\vec{q}} V(\vec{q}) e^{i \vec{q} \vec{\rho}}\left(b_{\vec{q}}+b_{-\vec{q}}^{+}\right),
$$

where $\vec{q}$ is the phonon wave vector: $\vec{q}\left(q_{x}, q_{y}\right)$.

The function $V(\vec{q})$ determines the phonon type with which an electron interacts. For interface symmetric or antisymmetric phonons $V(\vec{q})$ is expressed by

$$
\begin{gathered}
V_{I F}^{\{A\}}(\vec{q})=V_{I F}^{(1)}(\vec{q}) \cdot V_{I F}^{(2)}(\vec{q}, z)= \\
=\frac{C}{\sqrt{S}}\left[2 q\left[\frac{\partial}{\partial \omega}\left(\varepsilon_{c 1}\left\{\begin{array}{c}
\operatorname{th}\left(\varepsilon_{b 1} \frac{q L}{2}\right) \\
\operatorname{cth}\left(\varepsilon_{b 1} \frac{q L}{2}\right)
\end{array}\right\}-\varepsilon_{c 2}\right) \mid\right]^{-1 / 2} \times\right. \\
\times\left\{\begin{array}{c}
\left\{\begin{array}{c}
\operatorname{ch}\left(\varepsilon_{b 1} q z\right) \\
\operatorname{sh}\left(\varepsilon_{b 1} q z\right) \\
c h\left(\varepsilon_{b 1} \frac{q L}{2}\right)
\end{array}\right\},|z| \leq \frac{L}{2} \\
\left.\operatorname{sh}\left(\varepsilon_{b 1} \frac{q L}{2}\right)\right\} \\
\left\{\begin{array}{c}
1 \\
\operatorname{sgn}(z)
\end{array}\right\} \exp \left[-\varepsilon_{b 2} q\left(|z|-\frac{L}{2}\right)\right], \quad|z|>\frac{L}{2} .
\end{array}\right.
\end{gathered}
$$

The frequencies of symmetric (antisymmetric) phonons are determined from the dispersion relations:

$$
\varepsilon_{c 1}\left\{\begin{array}{c}
\operatorname{th}\left(\varepsilon_{b 1} \frac{q L}{2}\right) \\
c t h\left(\varepsilon_{b 1} \frac{q L}{2}\right)
\end{array}\right\}-\varepsilon_{c 2}=0
$$

within the frequency region when the inequalities are valid

$$
\begin{gathered}
\varepsilon_{1 z}(\omega) \varepsilon_{2 z}(\omega)<0, \varepsilon_{1 \perp}(\omega) \varepsilon_{1 z}(\omega)>0 \\
\text { i } \varepsilon_{2 \perp}(\omega) \varepsilon_{2 z}(\omega)>0 .
\end{gathered}
$$

In formulas (43), (44) the following notation is introduced: 


$$
\varepsilon_{c i}(\omega)=\sqrt{\varepsilon_{i \perp} \varepsilon_{i z}}, \quad \varepsilon_{b i}(\omega)=\sqrt{\varepsilon_{i \perp} / \varepsilon_{i z}}, \quad i=1,2, \ldots .
$$

For the confined phonons (symmetric and antisymmetric) $V(q)$ is

$$
\begin{aligned}
& V_{C}^{\left\{\begin{array}{l}
S A \\
A
\end{array}\right\}}(q)=V_{C}^{(1)}(\vec{q}) \cdot V_{C}^{(2)}(\vec{q}, z) \\
& \frac{C}{\sqrt{S}} \\
& \sqrt{\frac{\partial}{\partial \omega}\left(\varepsilon_{1 \perp} q^{2}+\varepsilon_{1 z} k_{m}^{2}\right) \frac{L}{2}-2 q \frac{\partial}{\partial \omega}\left\{\begin{array}{l}
\left.f_{S}(\omega) \cos \left(\frac{k_{m} L}{2}\right)\right) \\
f_{A}(\omega) \sin \left(\frac{k_{m} L}{2}\right)
\end{array}\right\}} \\
& \left\{\begin{array}{c}
\left\{\begin{array}{c}
\cos \left(k_{m} z\right) \\
\sin \left(k_{m} z\right)
\end{array}\right\}, \quad|z|<\frac{L}{2} \\
\left\{\begin{array}{c}
\cos \left(\frac{k_{m} L}{2}\right) \\
\operatorname{sgn}(z) \sin \left(\frac{k_{m} L}{2}\right)
\end{array}\right\} \exp \left[-\kappa_{2}\left(|z|-\frac{L}{2}\right)\right],|z|>\frac{L}{2},
\end{array}\right.
\end{aligned}
$$

where

$$
\begin{gathered}
\left\{\begin{array}{l}
f_{S}(\omega) \\
f_{A}(\omega)
\end{array}\right\}=\operatorname{sgn}\left(\varepsilon_{1 z}\right) \sqrt{-\varepsilon_{1 \perp}(\omega) \varepsilon_{1 z}(\omega) \times} \\
\times\left\{\begin{array}{l}
\sin \left(\frac{k_{m} L}{2}\right) \\
\cos \left(\frac{k_{m} L}{2}\right)
\end{array}\right\}-\operatorname{sgn}\left(\varepsilon_{2 z}\right) \varepsilon_{c 2}\left\{\begin{array}{l}
\cos \left(\frac{k_{m} L}{2}\right) \\
\sin \left(\frac{k_{m} L}{2}\right)
\end{array}\right\},
\end{gathered}
$$

$k_{m}$ is determined from

$$
\varepsilon_{1 z} k_{m}\left\{\begin{array}{l}
\sin \left(\frac{k_{m} L}{2}\right) \\
\cos \left(\frac{k_{m} L}{2}\right)
\end{array}\right\}-\varepsilon_{2 z} \kappa_{2}\left\{\begin{array}{c}
\cos \left(\frac{k_{m} L}{2}\right) \\
\sin \left(\frac{k_{m} L}{2}\right)
\end{array}\right\}=0,
$$

under the condition that

$\frac{\pi}{L}\left\{\begin{array}{c}2 m \\ 2 m-1\end{array}\right\}<k_{m}<\frac{\pi}{L}\left\{\begin{array}{c}2 m+2 \\ 2 m+1\end{array}\right\}$. For the symmetric phonons $(m=0,1,2, \ldots)$ and for antisymmetric phonons $(m=1,2,3, \ldots)$.

The semi-confined polarization phonons - their $E_{\perp}$ and $D_{z}$ being continuous functions at either interface with the properties similar to those of bulk crystal phonons - if $z= \pm \infty$, are characterized by the following $V(\mathrm{Q})$ for the symmetric, antisymmetric phonon modes:

$$
\begin{aligned}
& V_{H S}^{\left\{\begin{array}{l}
S \\
A
\end{array}\right\}}(\vec{Q})=V_{H S}^{(1)}(\vec{Q}) V_{H S}^{(1)}(\vec{Q}, z)= \\
& =\frac{C}{\sqrt{\tilde{L} S}}\left[\frac{\partial}{\partial \omega}\left(\varepsilon_{2 \perp} \sin ^{2} \theta_{2}+\varepsilon_{2 z} \cos ^{2} \theta_{2}\right)\right]^{-1 / 2} \frac{\sqrt{2}}{Q} \times \\
& \times\left[\varepsilon_{1 z}^{2} \kappa_{1}^{2}\left\{\begin{array}{l}
\operatorname{sh}^{2}\left(\frac{\kappa_{1} L}{2}\right) \\
\operatorname{ch}^{2}\left(\frac{\kappa_{1} L}{2}\right)
\end{array}\right\}+\varepsilon_{2 z}^{2} Q_{z}^{2}\left\{\begin{array}{l}
\operatorname{ch}^{2}\left(\frac{\kappa_{1} L}{2}\right) \\
\operatorname{sh}^{2}\left(\frac{\kappa_{1} L}{2}\right)
\end{array}\right\}\right]^{-1 / 2} \\
& \left\{\int \begin{array}{c}
1 \\
\operatorname{sgn}(z)
\end{array}\right\} \varepsilon_{1 z} \kappa_{1}\left\{\frac{\operatorname{sh}\left(\frac{\kappa_{1} L}{2}\right)}{\operatorname{ch}\left(\frac{\kappa_{1} L}{2}\right)}\right\} \times \\
& \times\left\{\begin{array}{c}
\times \sin \left(Q_{z}\left(|z|-\frac{L}{2}\right)\right)+ \\
+\varepsilon_{2 z} Q_{z}\left\{\frac{\operatorname{ch}\left(\frac{\kappa_{1} L}{2}\right)}{\operatorname{sh}\left(\frac{\kappa_{1} L}{2}\right)}\right\} \cos \left(Q_{z}\left(|z|-\frac{L}{2}\right)\right)
\end{array}\right\}, \\
& \varepsilon_{2 z} Q_{z}\left\{\begin{array}{l}
\operatorname{ch}\left(\kappa_{1} z\right) \\
\operatorname{sh}\left(\kappa_{1} z\right)
\end{array}\right\}, \quad|z|<\frac{L}{2} \\
& |z|>\frac{L}{2} \text {. }
\end{aligned}
$$

where the angle $\theta_{2}$ is the one between the phonon wave vector $\overrightarrow{\mathrm{Q}}=\left(\vec{q}, \mathrm{Q}_{z}\right)$ and the z-axis.

Eqs. (46) are valid when the inequality $\varepsilon_{2 \perp} \varepsilon_{2 z}>0$ is satisfied, because only in this case vibrations will decay in the nanofilm region since $\kappa_{2}=\sqrt{\frac{\varepsilon_{2 \perp}(\omega)}{\varepsilon_{2 z}(\omega)}} q$.

Another type of vibrations - the ones that spread in the medium - can exist only when the two inequalities are fulfilled:

$$
\varepsilon_{1 \perp}(\omega) \varepsilon_{1 z}(\omega)<0, \quad \varepsilon_{2 \perp}(\omega) \varepsilon_{2 z}(\omega)<0 .
$$

The analysis shows that there is no frequency region in the AlN / GaN / AlN heterosystem where inequalities (47) hold, therefore the above-mentioned phonons do not exist in the heterosystem under consideration [9].

In order to determine the polaron energy, one must solve the Schrцdinger equation with Hamiltonian (34):

$$
\hat{H}_{e} \psi_{e}(\vec{r})=E_{n}(\vec{k}) \psi_{e}(\vec{r}) .
$$

The electron potential energy in the heterosystem is chosen as a rectangular potential well 


$$
V(z)=\left\{\begin{array}{cc}
0, & |z|<\frac{L}{2} \\
V_{0}, & |z| \geq \frac{L}{2}
\end{array} .\right.
$$

Then the wave function looks as follows:

$$
\psi_{e}(\vec{r})=\frac{1}{\sqrt{S}} e^{i \vec{k} \vec{\rho}} \varphi_{n}(z),
$$

where $\varphi_{n}(z)=\left\{\begin{array}{c}A e^{\chi_{0} z}, \quad z<-L / 2 \\ \alpha \sin (\chi z)+\beta \cos (\chi z), \quad|z|<L / 2, \\ B e^{-\chi_{0} z}, \quad z>L / 2\end{array}\right.$

$$
\chi_{0}=\sqrt{\frac{2 m_{\| 2}}{\hbar^{2}}\left(V_{0}-\tilde{E}_{n}\right)}, \chi=\sqrt{\frac{2 m_{\| 1}}{\hbar^{2}} \tilde{E}_{n}},
$$

and the electron energy is described by

$$
E_{n}(\vec{k})=\frac{p^{2}}{2 m_{\perp}}+\tilde{E}_{n},
$$

$\vec{p}$ is the electron impulse, and $\tilde{E}_{n}$ is found from the dispersion relation which is a result of continuity condition of the wave function and its probability current density at the interfaces.

To determine the polaron energy, the perturbation theory and the variational Lee-Low-Pains (LLP) method are used [19, 20]. By applying the LLP method, it is taken into account that the considered system contains a fast and a slow subsystems. The electron motion in the direction normal to the interface is assumed a fast subsystem. The adiabatic approximation is therefore used, Hamiltonian (33) is averaged over the functions of the electron ground stationary state in the motion along the z-axis:

$$
\begin{gathered}
\hat{H} e f=\left\langle\varphi_{1}(z)|\hat{H}| \varphi_{1}(z)\right\rangle= \\
=\tilde{E}_{1}+\frac{p^{2}}{2 m_{\perp}}+\hat{H} p h+\sum_{\vec{q}} V^{(1)}(\vec{q})\left(e^{i \vec{q} \vec{\rho}_{M}} b_{\vec{q}}+\text { e.c. }\right),
\end{gathered}
$$

where $M_{1 n}=\int_{-\infty}^{\infty} \varphi_{1}^{*}(z) V^{(2)}(\vec{q}, z) \varphi_{n}(z) d z, n=1$.

Two unitary transformations are consecutively applied to Hamiltonian (20) by the operators

$$
\begin{gathered}
\hat{S}=\exp \left[\frac{i}{\hbar}\left(\vec{P}-\sum_{\vec{q}} b_{\vec{q}}^{+} b_{\vec{q}} \hbar \vec{q}\right) \vec{\rho}\right], \\
\hat{U}=\exp \left[\sum_{\vec{q}}\left(b_{\vec{q}}^{+} f(\vec{q})-b_{\vec{q}} f^{*}(\vec{q})\right)\right],
\end{gathered}
$$

where $\vec{P}=\hbar \vec{k}$ is the polaron impulse.
After averaging the obtained expression over the vacuum phonon state and minimizing the functional over $f(\vec{q})$ and $f^{*}(\vec{q})$, the polaron energy of the heterosystem is found [21-23]:

$$
\begin{gathered}
E_{p o l}(\vec{k})=\tilde{E}_{1}+\frac{\hbar^{2} k^{2}}{2 m_{\perp}}\left(1+\eta^{2}\right)+ \\
+\sum_{\vec{q}} \frac{-2 \cdot\left|V^{(1)}(\vec{q})\right|^{2}\left|M_{11}\right|^{2}}{\hbar \omega-\frac{\hbar \vec{q} \vec{P}}{m_{\perp}}(1-\eta)+\frac{\hbar^{2} q^{2}}{2 m_{\|}}}+ \\
\sum_{\vec{q}} \frac{\left|V^{(1)}(\vec{q})\right|^{2}\left|M_{11}\right|^{2}\left\{\hbar \omega-\frac{\hbar \vec{q} \vec{P}}{m_{\perp}}+\frac{\hbar^{2} q^{2}}{2 m_{\perp}}\right\}}{\left\{\hbar \omega-\frac{\hbar \vec{q} \vec{P}}{m_{\perp}}(1-\eta)+\frac{\hbar^{2} q^{2}}{2 m_{\perp}}\right\}^{2}},
\end{gathered}
$$

where $\eta \vec{P}=\sum_{\vec{q}}\left|f_{\min }(\vec{q})\right|^{2} \hbar \vec{q}$,

$$
f_{\min }(\vec{q})=-\frac{V^{(1)^{*}}(\vec{q}) M_{11}^{*}}{\hbar \omega+\frac{\hbar^{2} q^{2}}{2 m_{\perp}}-\frac{\hbar^{2}}{m_{\perp}} \vec{k} \vec{q}(1-\eta)} .
$$

The polaron energy in the bulk material is determined by similar formulas [19]. The difference is that expression (52) contains the function $M_{11}$, resulting from the translational symmetry deviation of the system in the z-direction.

The crystals $A l N, G a N$ are characterized by a small constant of the electron-phonon interaction ( $\alpha=0.681, \alpha=0.466$ accordingly), therefore to determine the polaron energy, one can use the perturbation theory [24, 25]:

$$
\begin{gathered}
E_{p o l}(\vec{k})=\tilde{E}_{1}+\frac{\hbar^{2} k^{2}}{2 m_{\perp}}+ \\
+\sum_{n, \vec{q}} \frac{\left|V^{(1)}(\vec{q})\right|^{2}\left|M_{1 n}\right|^{2}}{\tilde{E}_{1}-\tilde{E}_{n}-\hbar \omega+\frac{\hbar^{2} k^{2}}{2 m_{\perp}}-\frac{\hbar^{2}(\vec{k}-\vec{q})^{2}}{2 m_{\perp}}} .
\end{gathered}
$$

In receiving formula (53), the adiabatic approach was not used, thus it can be applied not only for small values $L$ of quantum well widths. Expressions (52) and (53) reflect the wave-vector dependence of the polaron energy - the quasiparticle dispersion relation. 


\section{Discussion of the results}

The specific calculations are performed for the heterostructures $A l N / G a N / A l N$, вакуум/GaN/AlN, $\mathrm{GaN} / \mathrm{ZnO} / \mathrm{AlN}$. Material parameters are taken to be: $\varepsilon^{\infty}=5.29, \omega_{L t}=743 \mathrm{sm}^{-1}, \omega_{L z}=735 \mathrm{sm}^{-1}$, $\omega_{T t}=561 \mathrm{sm}^{-1}, \omega_{T z}=533 \mathrm{sm}^{-1}$ forGaN; $\varepsilon^{\infty}=4.68$, $\omega_{L t}=916 \mathrm{sm}^{-1}, \omega_{L z}=893 \mathrm{sm}^{-1}, \omega_{T t}=673 \mathrm{sm}^{-1}$, $\omega_{T z}=660 \mathrm{sm}^{-1}$ for $A l N ; \varepsilon^{\infty}=3.7, \omega_{L t}=578 \mathrm{sm}^{-1}$, $\omega_{L z}=594 \mathrm{sm}^{-1}, \omega_{T t}=381 \mathrm{sm}^{-1}, \omega_{T z}=409 \mathrm{sm}^{-1}$ for $Z n O$ [7]. Unlike the case of heterostructures of isotropic crystals, there appears a number of peculiarities of optical phonon spectrum. The main reason of this result is found in dispersion relation (5) from which, generally speaking, one cannot separate the wave vector of vibration frequency. In addition, if we take account of the conditions at the interfaces, we obtain finite energy integrals for different mode phonons. By comparing theory and experiment, these regions of phonon frequencies make it possible to determine composition of the nanoheterostructure.

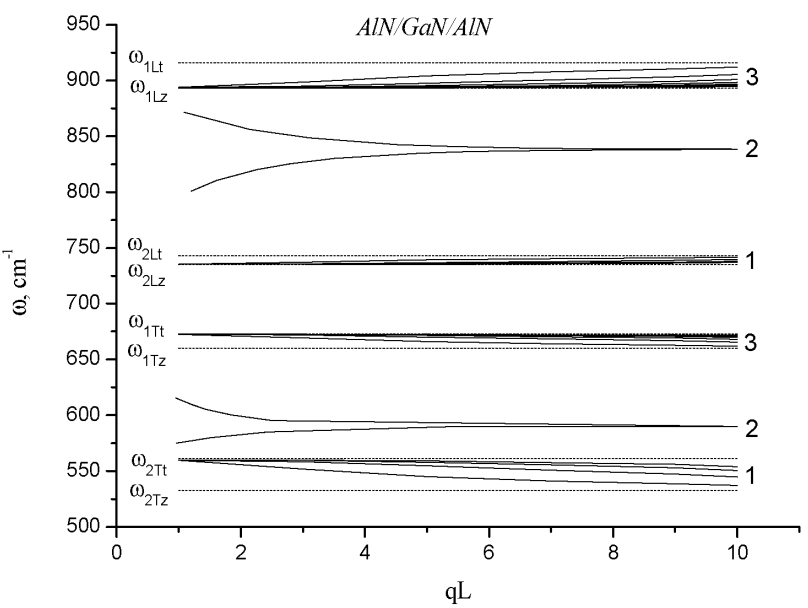

Fig. 2. The dispersion relation of polarization optical phonons in a quantum well $A l N / G a N / A l N$. Confined (1), interface (2) and half-space (3) phonon modes in the given intervals of phonon frequencies are presented. Characteristic frequencies are indicated with dashed curves.

Fig. 2 presents the dispersion relations of confined, interface and half-space polarization phonons. It is seen that every phonon mode exists in two frequency regions. For confined phonons of the heterostructure $\mathrm{AlN} / \mathrm{GaN} / \mathrm{AlN}$ frequency intervals are: $\omega_{2 T z} \leq \omega_{I}^{c} \leq \omega_{2 T t}, \quad \omega_{2 L z} \leq \omega_{I I}^{c} \leq \omega_{2 L t}$. Interface phonons exist at the frequencies : $\omega_{2 T t} \leq \omega_{I}^{i} \leq \omega_{1 T z}$, $\omega_{2 L t} \leq \omega_{I I}^{i} \leq \omega_{1 L z}$, and half-space phonons $\omega_{1 T z} \leq \omega_{I}^{h} \leq \omega_{1 T t}, \quad \omega_{1 L z} \leq \omega_{I I}^{h} \leq \omega_{1 L t}$. Unlike in het- erostructures of cubic lattice structure crystals the frequency of confined phonons is not constant, it is a monotonous function of the wave vector and also depends on the number $m$. In particular, in the region $\omega_{I}^{c}$ the least value of $m(m=0$ for antisymmetric modes) is related to the mode with the smallest, and in the region $\omega_{I I}^{c}$ with the largest frequency. At the given wave vector the rise of $m$ results in the reduction (interval $\omega_{I I}^{c}$ ) or in the increase (interval $\omega_{I}^{c}$ ) of frequency. There are four interface phonon modes in two regions of frequencies. For small values of the wave vector they sufficiently differ in frequency. The increase of $q L$ results in the fact that two modes in pairs degenerate into one also at great values of $q L$. Similar dependences are received for half-space phonons.

The data of calculations of the confined and interface phonon spectrum of asymmetric nanoheterostructures vacuum/GaN/AlN, GaN/ZnO/AlN are given in Fig. 3(a,b). Because of more rigid conditions applied on the existence of interface phonons, the number of these phonon modes decreases compared with a symmetric heterosystem. Alongside, the degeneracy of the spectrum is lifted at large values of the wave vector. As to confined phonons, the heterosystems differ not only in frequency numerical values but also in the dependence $\omega=\omega(q)$. For both frequency intervals the dispersion relations of confined phonons of the heterosystem vac$u u m / G a N / A l N$ are qualitatively identical to those of the structure $A l N / G a N / A l N$. In the heterostructure $\mathrm{GaN} / \mathrm{ZnO} / \mathrm{AlN}$ these dependences are slightly different: the rise of $q L$ is followed by the increase of frequencies for "lower" interval, and by their decrease for "upper" frequency region. The analysis of existence conditions of propagating phonons shows that in the heterosystems under consideration they do not exist.

In the investigation of polaron states, we consider the region of small values of wave vectors ( $k \approx 0)$. The quantities $\eta(\vec{k})$ and $E_{\text {pol }}(\vec{k})$ are obtained by expanding the corresponding expressions in series and restricting to first terms of the expansion [19]. Regarding the polaron dispersion relation in a small but finite region of the wave-vector change $0 \leq k<k_{f}, k_{f}=\sqrt{\frac{2 m_{\perp} \omega}{\hbar}}<<k_{0}$, where $k_{0}$ is the wave vector critical value in the Brillouin zone, the calculations $E_{\text {pol }}=E_{\text {pol }}(\vec{k})$ in this report have been carried out without any additional simplification of formulas (52), (53). 

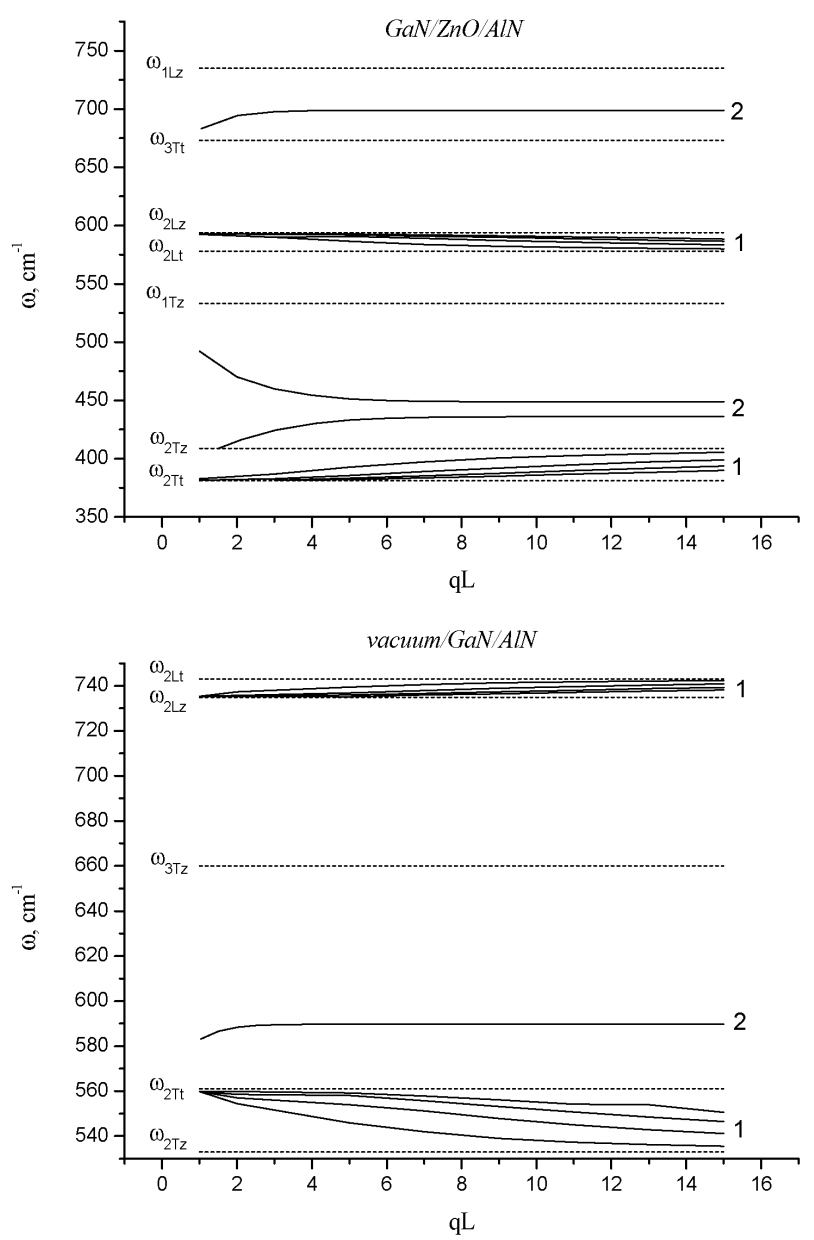

Fig. 3. The dispersion relation of polarization optical phonons in quantum wells $G a N / Z n O / A l N$ (a) and vacuum/GaN/AlN (b): confined (1) and interface (2) phonon modes in the given intervals of phonon frequencies. Characteristic frequencies are indicated with dotted curves.

In Fig.4 the dependence of the polaron binding energy of the heterosystem $A l N / G a N / A l N$ on the nanofilm thickness is presented. For the comparison the polaron binding energies of bulk crystals $G a N$ and $A l N$ are given. It is seen that the polaron binding energy $\left(E_{c} \equiv\left|E_{p o l}(0)\right|\right)$ in a bulk crystal $G a N$ is smaller than that in the heterosystem. The reduction of quantum well width $(L)$ leads to the increase of this energy and the rise of the dispersion relation deformation.

As to partial contributions, the confined phonons are predominant at $L>40 \AA$, though one cannot neglect interface phonon contributions in the range of $40 \AA<\mathrm{A}<100 \AA$. For thicknesses $L<30 \AA$ the interface phonon contribution becomes greater than that of the confined phonons. As to the halfspace phonon contribution, the calculations show that they have little effect on the polaron binding energy. For illustration, at $L=25 \AA$ for the $A l N /$ $\mathrm{GaN} / \mathrm{AlN}$ double heterostructure within the infinite quantum well (IQW) model, the contribution makes $22.536 \mathrm{meV}$ in the case of confined phonons, for interface phonons $22.633 \mathrm{meV}$, and half-space phonons $2.035 \mathrm{meV}$, while at $L=100 \mathrm{~A}$ it is 32.484 $\mathrm{meV}, 8.711 \mathrm{meV}, 1.184 \mathrm{meV}$ respectively. The comparison of two models - the IQW $(V=\infty$ in (49)) and the finite quantum well (FQW) at the interface of the heterosystem - shows similar results for all phonon types not only for great $L$ because of significant real quantum well depth. The difference becomes essential (particularly for the confined phonons at $L<60 \AA$ (e.g., if $L=50 \AA$, it makes $17 \%)$ ).

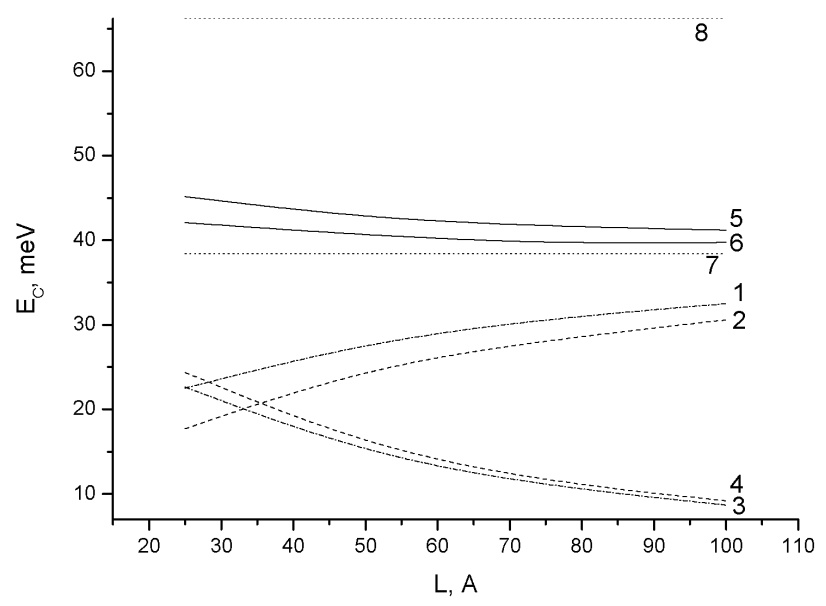

Fig.4. The polaron binding energy of the bulk crystals $G a N(7), A l N(8)$ and heterosystem $A l N / G a N / A l N$ in case of IQW (curves 1,3,5), FQW (curves 2,4,6). 1,2 - confined phonons are taken into account, 3,4 - interface phonons are regarded, 5,6 - all phonons are taken into account.

Fig. 5 shows the dependence of the dispersion relation of a polaron that moves parallel to the heterosystem interfaces at $L=25 \AA$. In the range of small wave-vector values for all phonon modes and both potential well models, the quadratic dependences of functions $E_{p o l}=E_{p o l}(k)$ are received. The wave-vector value rise results in the "dispersion relation deformation". If one takes account of all phonon modes, as is seen from the figure, the polaron energy for the $V_{0}=\infty$ model is smaller than that for the finite value of $V_{0}$. However, the wave-vector growth is followed by the reduction of the polaron energy difference within different QW models. 


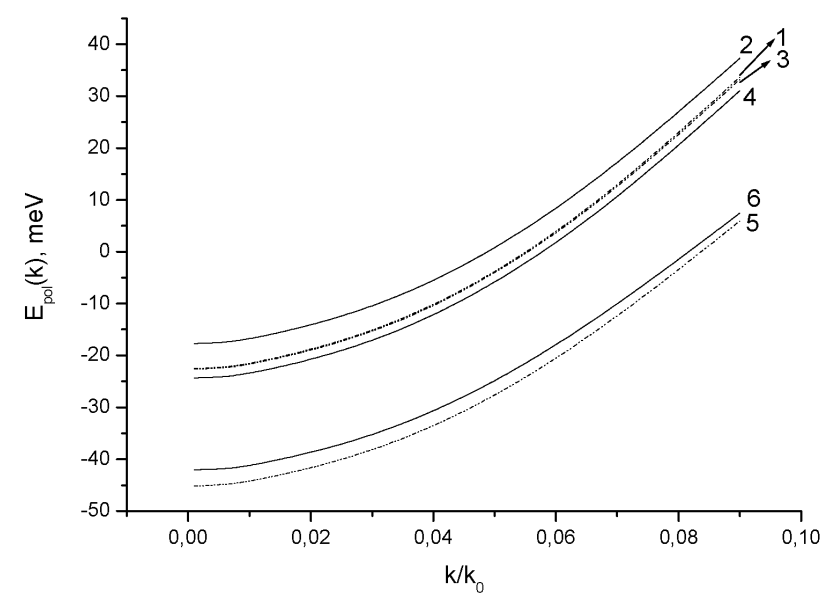

Fig.5. Polaron energy of the heterosystem $A l N / G a N / A l N$ for QW at $L=25 \mathrm{~A}$ in the cases of IQW (curves $1,3,5$ ) and FQW (curves 2,4,6). 1,2 show the energy with regard for confined phonons, 3,4 stand for the interface phonons, 5,6 indicate all phonons.

Since the dispersion relation is known, one can determine the polaron average speed:

$$
\vec{v}=\frac{1}{\hbar} \nabla_{\vec{k}} E_{\text {pol }}(\vec{k}) \text {. }
$$

This quantity enters the formulas of kinetic coefficients as well as defines the degree of polaron dispersion relation "deformation". It is seen from Fig. 6 that in the region of large wave-vector values $k \approx k_{f}$, the increase of $k-$ as a result of all phonon modes contribution - leads to $v=v(k)$ approaching saturation, i.e., the polaron dispersion relation $E_{p o l}=E_{p o l}(k)$ transfers from a quadratic function (at $k=0$ ) into a liner one (at $k=k_{f}$ ).

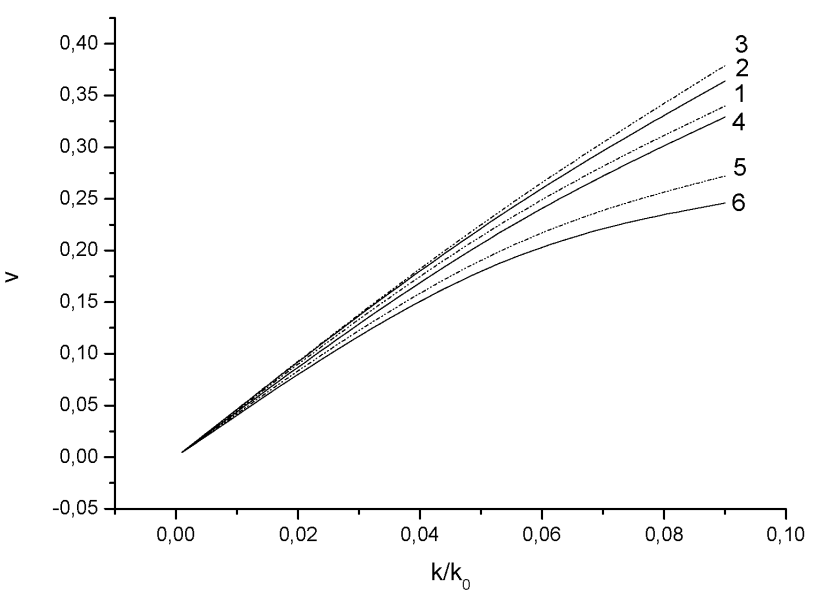

Fig. 6 Polaron average speed of the $A l N / G a N / A l N$ heterosystem for QW with $L=25 \mathrm{~A}$ in the cases of IQW (curves 1,3,5) and FQW (curves 2,4,6). 1,2 regard for the interaction with confined phonons, 3,4 account for interface phonons, 5,6 regard for all phonons.
Alongside with the speed, the polaron effective mass is also important:

$$
\frac{1}{m_{p}}=\left.\frac{1}{\hbar^{2}} \frac{\partial^{2} E_{p o l}(k)}{\partial k^{2}}\right|_{k=0} .
$$

Fig. 7 shows that the reduction of $L$ is followed by polaron effective mass growth. It points out - similarly to the polaron binding energy enhancement - the increase of the effective electronphonon interaction. The rise of particle spatial confinement also leads to its effective mass growth.

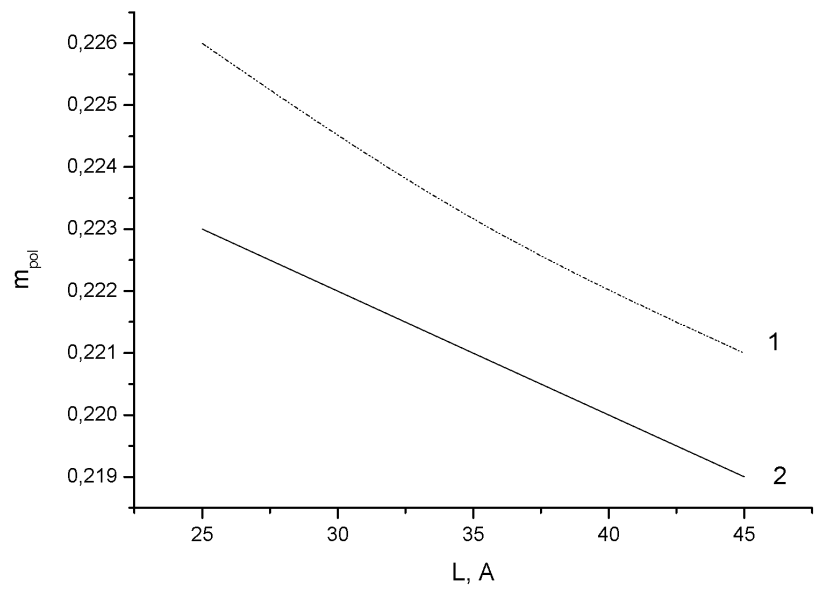

Fig.7 Polaron effective mass of the $A l N / G a N / A l N$ heterosystem in the cases of IQW (1) and FQW (2) with regard for all phonons.

Therefore, the article presents wave vector - dependent energies of confined, interface, half-space, and propagating phonons for symmetric and asymmetric three-layer nanoheterosystems of hexagonal lattice structure crystals. The specific calculations are performed for three-layer symmetric $(\mathrm{AlN} / \mathrm{GaN} /$ $A l N)$, and three-layer asymmetric heterosystems (vacuum $/$ GaN/AlN, GaN/ZnO/AlN). It is defined and analysed the character of frequency dependences of every phonon mode type as a function of wave vector. The report also presents a theoretical study of the polaron dispersion relation in the $\mathrm{GaN}$ crystal and $\mathrm{AlN} / \mathrm{GaN} / \mathrm{AlN}$ double nanoheterostructure by taking into account all types of polarization oscillations with which an electron interacts within the models of infinite and finite barriers. Calculations of the average speed and polaron effective mass are performed. It is shown that, the interface phonon contribution in the polaron energy decreases with nanofilm thickness increase, while that of the confined phonons grows. The calculation data of the polaron dispersion relation in the region $k<k_{f}$ in 
different directions of the polaron wave vector with respect to the $C$-axis of the crystal are given. The reduction of the dimension number of the system, increase of the wave-vector $k$ cause an effective enhancement of the electron-phonon interaction.

\section{References}

1. R. Loudon. The Raman effect in crystals // $A d v$. Phys., 50(7), pp.813-864 (2001).

2. K. Huang and B.F. Zhu // Phys. Rev. B., 38(18), pp.13377-13386 (1988-II).

3. H. Rucher, E. Molinari and P. Lugli. // Phys. Rev. B., 44, p.3463 (1991).

4. A.K. Sood, J. Menendez, M. Cardona and K. Ploog. // Phys. Rev. Lett., 54, p.2115 (1995).

5. R. Hessmer, A. Huber, T. Egeler, M. Haines, G. Trankle, G. Weiman and G. Abstreiter. // Phys. Rev. B., 46, p.4071 (1992).

6. S. Nakamura and S.F. Chichibu. Introduction to Nitride Semiconductor Blue Lasers and Light Emitting Diodes. Taylor and Francis, London, 890p (2000)

7. B.C. Lee, K.W. Kim, M.A. Stroscio and M. Dutta // Phys. Rev. B., 58(8), pp.4860-4865 (1998).

8. E.R. Racec and D.E. Brancus // J. Phys.: Condens. Matter., 10, p.3845 (1998).

9. S.M. Komirenko, K.W. Kim, K.M. Stroscio and M. Dutta. Dispersion of polar optical phonons in wurtzite quantum wells // Phys. Rev. B., 59(7), pp.50135020 (1999).

10. J. Gleize, M.A. Renucci, J. Frandon and F. Demangeot // Phys. Rev. B., 60(23), pp.15985-15992 (1999).
11. J.-J. Shi // Phys. Rev. B., 68, pp.165335-1 - 16533511 (2003).

12. J.-J. Shi, X.-L. Chu and E.M. Goldys // Phys. Rev. B., 70, pp.115318-1 - 115318-8 (2004).

13. J. Gleise, J. Frandon, F. Demangeot, M.A. Renucci, C. Adelmann, B. Dandin, G. Feuillet, B. Damilano, N. Grandjean and J. Massies // Appl. Phys. Lett., 77, p.2174 (2000).

14. J. Zhang, X.S. Peng, X.S. Wang, Y.W. Wang and L.D. Zhang// Chem. Phys. Lett., 345, p.372 (2001).

15. H.L. Liu, C.C. Chen, C.T. Chin, C.C. Yeh, C.H. Chen, M.Y. Yu, S. Keller and S.P. Den Baars // Chem. Phys. Lett., 345, p.245 (2001).

16. H.J. Choi, J.C. Johnson, R. He, S.K. Lee, F. Kim, P. Panzanskie, J. Goldberger, R.J. Saykally and P. Yang // J. Phys. Chem. B., 107, p.8271 (2003).

17. N. Mori, T. Ando // Phys.Rev.B., 40(9), pp.61756788 (1989-II).

18. M.E.Mora-Ramos // Phys. Status Solidi B. 223, 843 (2001).

19. Lee T.D., Low F.E. and Pines D // Phys. Rev. 90, 297 (1953).

20. Firsov Yu.A. Polyarony. M., Nauka (1975) (in Russian).

21. V.I. Boichuk, V.A. Borusevych // Ukr. J. Phys. 51, 168 (2006).

22. V.I. Boichuk, V.A. Borusevych // J. Phys. Studies 10, 39 (2006).

23. V.I. Boichuk, V.A. Borusevych, I.P. Kogoutiouk // Condens. Matter Phys. 10, 219 (2007).

24. M.E.Mora-Ramos, F.J.Rodriguez and L.Quiroga // J. Phys.: Condens. Matter 11, 8223 (1999).

25. M.E.Mora-Ramos // Phys. Status Solidi B. 219, R1 (2000) 